\title{
Comparison of Urine Dipstick Nitrite Test with Urine Culture in the Diagnosis of Urinary Tract Infection
}

\author{
Sanjib Adhikari, ${ }^{1,2}$ Sanjeep Sapkota, ${ }^{1}$ Prativa Poudel, ${ }^{1,2}$ Ramesh Sharma Regmi, ${ }^{1,2}$ Sujata Kafle, ${ }^{1}$ \\ Sangita Baral, ${ }^{1}$ Nirajan Dallakoti, ${ }^{1}$ Jid Chani Rana, ${ }^{3}$ Bipin Mahato, ${ }^{1}$ Komal Raj Rijal ${ }^{2}$ \\ 'Department of Microbiology, Birendra Multiple Campus, Tribhuvan University, Bharatpur, Chitwan, Nepal, \\ ${ }^{2}$ Central Department of Microbiology, Tribhuvan University, Kirtipur, Kathmandu, Nepal, ${ }^{3}$ Department of \\ Microbiology, Bharatpur Hospital, Bharatpur, Chitwan, Nepal.
}

\section{ABSTRACT}

\section{Introduction}

Urine dipstick is a rapid, cost-effective test used as a marker for quick detection of bacterial Urinary Tract Infection (UTI). Nitrite test depends on the conversion of nitrate to nitrite by the action of Gramnegative bacteria present in urine. The purpose of this study was to determine the sensitivity, specificity, positive predictive value and negative predictive value of Nitrite test in relation to urine culture.

\section{Methods}

Two hundred fresh uncentrifuged urine samples with suspicion of UTI were collected and tested for nitrite by using urine dipstick strip (COMBI-10SL) prior to the culture. Nitrite was considered as positive if there was a change in color of dipstick from colorless towards pink within 60 seconds. Quantitative urine culture was performed by using the strips calibrated to deliver $0.02 \mu \mathrm{L}$ of urine on Cystine Lactose Electrolyte Deficient (CLED) agar. All plates were incubated at $37^{\circ} \mathrm{C}$ and read after 24 to 48 hours. Culture was considered as the gold standard to evaluate the performance of the dipstick test. Antimicrobial susceptibility testing (AST) of the isolates was done using the Kirby-Bauer disc diffusion method following Clinical and Laboratory Standards Institute (CLSI) guidelines.

\section{Results}

Out of 200 samples, 36 (18.00\%) samples showed significant growth whereas 164 (82.00\%) samples did not show significant growth. Out of 36 samples, 25 (69.44\%) samples showed positive nitrite test while 11 (30.55\%) showed negative nitrite test. The sensitivity, Specificity, Positive Predictive Value and Negative Predictive Value of the nitrite test were $69.44 \%, 89.63 \%, 59.52 \%$ and $93.03 \%$ respectively. The most frequently isolated bacteria were Escherichia coli 29 (80.55\%) followed by Enterobacter aerogenes $3(8.33 \%)$ and Pseudomonas aeruginosa 2 (5.56\%). The antimicrobial profile of the isolates revealed that $14(38.89 \%)$ isolates were MDR.

\section{Conclusions}

Dipstick test for the detection of nitrite in urine is sensitive and specific and can be used for the primary screening of UTI in a resource-limited setup. Confirmatory diagnosis should be followed by the urine culture method.

Keywords: MDR, nitrite test, urine dipstick, UTI.

Correspondence: Mr. Sanjeep Sapkota, Department of Microbiology, Birendra Multiple Campus, Tribhuvan University, Bharatpur, Chitwan, Nepal. E-mail: sansanjeep123@gmail.com. Phone: +977-9845529276. 


\section{INTRODUCTION}

Urinary tract infection (UTI) is defined as the presence of multiplying bacteria within the urinary tract. The presence of a significant number of bacteria in aseptically collected urine is an indication of urinary tract infection. Despite the presence of different host defense mechanisms against microbial infection in the urinary tract, it is an important health problem affecting millions of people annually from all age groups. ${ }^{1}$

The urinary tract consists of the kidney, ureter, bladder and urethra. All of the urinary tract above urethra in a healthy human is sterile. Thus, urine is a sterile body fluid that gets contaminated as it passes through urethra. UTI is among the most frequently acquired infection in the community, but also in hospitals and other health care institutions, causing a huge amount of antibiotic treatment calling for an update of current trends. ${ }^{2}$ UTI is caused by the presence of bacteria in urine, although fungi and viruses may be involved. Most often the bacteria involved in UTI are Escherichia coli, Klebsiella spp., Citrobacter spp., Proteus spp., Staphylococcus saprophyticus, Staphylococcus aureus, coagulasenegative Staphylococcus (CoNS) and betahemolytic Streptococci. Among these, E. coli is the most predominant uropathogens accounting for $80-85 \%$ cases. $^{3,4}$

In more complicated UTI, particularly in recurrent infections, the relative frequency of infection caused by Proteus, Pseudomonas, Klebsiellaand Enterobacter spp. increases. Hospitalized patients are more likely to be infected by E. coli, Klebsiella spp., Proteus mirabilis and Enterobacteriaceae. In addition, UTIs are the leading cause of Gram-negative sepsis in hospitalized patients and are the origin of about half of all nosocomial infections caused by urinary catheters. ${ }^{5}$ Candida urinary infection is usually found in diabetic patients and those with immune suppression. ${ }^{6}$ Risk factor includes female anatomy, sexual intercourse, diabetes, obesity and family history. ${ }^{7}$

Many prompt diagnostic methods are available including wet-mount microscopy, Gram stain, dipstick and automated assays, but the gold standard method for diagnosis of UTI is quantitative urine culture. ${ }^{8}$ Urine culture is an expensive procedure and needs a well-equipped microbiology laboratory with experienced technicians. ${ }^{9}$

A dipstick nitrite test is sensitive and specific when properly used forfirst-morning urine because bladder incubation for the organism to convert nitrate to nitrite takes a minimum of four hours. ${ }^{10}$ So random specimens collected at any time and urine from patients with draining catheters do not show a good correlation between significant bacteriuria and nitrite test. False positive results commonly occur with poorly collected or stored specimens resulting in contamination and post collection bacterial proliferation. False negative results may be due to low $\mathrm{pH} \quad(<6)$, ascorbic acid or urobilinogen. ${ }^{11}$

Rapid diagnostic tests can rule out urine infection, are inexpensive, less time-consuming and are useful in small laboratories having no culture facility. They are also more rapid than culture in diagnosing UTI. A sterile urine sample is not required for dipstick test, therefore, it is easy to collect samples especially in children by a noninvasive method. ${ }^{12}$ For dipstick method of diagnosing UTI, there is no requirement of trained staff and well-equipped laboratory. ${ }^{13}$ Due to the clinical significance of early diagnosis, rapid urine tests such as urine dipsticks are used widely. ${ }^{14}$ In recent times, antimicrobial resistance among clinical isolates is ever-increasing and posing public health threat. ${ }^{15}$ UTI is often treated 
with different broad-spectrum antibiotics when one with a narrow spectrum of activity may be appropriate because of concerns about infection with resistant organisms. ${ }^{16}$ Culture of urine for specific bacteria followed by antibiogram testing is still regarded as the best way to diagnose UTI. ${ }^{17}$

The major objective of the study was to evaluate the accuracy of dipstick nitrite tests for rapid screening of urine samples, keeping semi quantitative culture as the gold standard for the diagnosis of UTI. In addition, the study also investigated the common uropathogens and antimicrobial susceptibility patterns of the isolates recovered from the patients visiting Bharatpur Hospital.

\section{METHODS}

The study was a descriptive study carried out prospectively at the Microbiology Laboratory of Bharatpur Hospital, Chitwan. The study was conducted over a period of three months, from November 2018 to January 2019. A total of 200 samples were collected from patients suspected of UTI. Patients of all age groups and sex were included in the study. All midstream urine samples received in the Microbiology laboratory for culture were recruited in the study, whereas the samples showing insignificant growth, mixed growth, samples from the patients who had taken antibiotics before 72 hours or had indwelling Foley Catheters were discarded. Samples with improper labeling and inappropriate collection methods were also rejected. Clean catch, MidStream Urine (MSU) samples with suspicion of UTI were collected in a sterile,wide-mouthed bottle and tested for nitrite by using dipstick nitrite test strip (COMBI-10SL, UK). Nitrite was considered as positive if there was a change in color of dipstick from colorless towards pink within 60 seconds. Urine culture was performed by using the strips calibrated to deliver $0.02 \mu \mathrm{L}$ of urine on Cysteine Lactose Electrolyte Deficient (CLED) agar. All plates were incubated at $37^{\circ} \mathrm{C}$ and read at 24 and 48 hours. Culture was considered as the gold standard to assess the performance characteristic of dipstick test. Obtained isolates were analyzed for the antimicrobial resistance pattern. For this, a panel of 10 antibiotics [Amikacin (30 $\mu g$ ) Amoxyclav (30 $\mu g$ ) Ciprofloxacin $(5 \mu \mathrm{g})$ Cefixime $(30 \mu \mathrm{g})$ Levofloxacin $(5 \mu \mathrm{g})$ Nalidixic acid $(30 \mu g)$ Nitrofurantoin (300 $\mu \mathrm{g})$ Cotrimoxazole $(25 \mu \mathrm{g})$ Gentamicin (10 $\mu \mathrm{g})$ Cefalexin $(30 \mu \mathrm{g})$ Polymyxin-B (300 units) Colistin $(10 \mu \mathrm{g})]$ were used. Antibiotic sensitivity testing was performed by Kirby Bauer disc diffusion method and interpreted as per CLSI guidelines (2016). ${ }^{18}$ The turbidity of the inoculum was adjusted to the equivalent turbidity of $0.5 \mathrm{McF}$ arland standards. Eighteen hours cultures of test organisms incubated at $37^{\circ} \mathrm{C}$ were standardized by diluting to 0.5 McFarland turbidity standards before spreading over the surface of Mueller-Hinton agar (Titan Biotech Ltd., Bhiwadi-301019, Rajasthan, India) plates using a sterile cotton swab and allowed to dry for 2 to 5 minutes. By using sterilize tweezers, antibiotic discs were placed $2 \mathrm{~cm}$ apart from each other. The plates were incubated at $37^{\circ} \mathrm{C}$ for 24 hours. Following incubation, the diameter of the inhibition zone was measured with a transparent ruler and expressed in millimeters $(\mathrm{mm})$ as sensitive, intermediate and resistant based on CLSI guidelines. Resistance to at least one drug from 3 different antibiotics of different structural classes was considered MDR as described elsewhere. ${ }^{19}$ Data were analyzed by using Statistical Package for Social Sciences (SPSS) version 16. Diagnostic measures i.e., sensitivity, specificity, PPV and NPV were calculated by standard formulae using culture as gold standard. 


\section{RESULTS}

\section{Growth pattern}

Out of 200 mid-stream urine samples, 36 $(18 \%)$ samples showed significant growth whereas 164 (82\%) samples did not show significant growth. Positive samples showed the growth of different Gram-negative organisms.

\section{Comparison between nitrite test and culture}

Out of total samples ( $n=200), 36$ samples showed significant growth of which 25 samples showed nitrite test positive while 11 showed nitrite test negative. Among total of 42 nitrite test positive samples, 17 growth negative samples showed nitrite test positive while 147 growth negative samples showed nitrite test negative. The sensitivity and Specificity of the nitrite test were $69.44 \%$ and $89.63 \%$. Also, positive predictive value and negative predictive value of nitrite test were $59.52 \%$ and $93.03 \%$ respectively considering culture as the gold standard (Table 1).

Table 1. Comparison between nitrite test and culture

\begin{tabular}{|l|l|l|l|}
\hline Nitrite test & Growth & No Growth & \multicolumn{1}{|c|}{ Total } \\
\hline Positive & 25 & 17 & 42 \\
\hline Negative & 11 & 147 & 158 \\
\hline Total & 36 & 164 & 200 \\
\hline
\end{tabular}

Sensitivity: $69.44 \%$

Specificity: $89.63 \%$

Positive predictive value: $59.52 \%$

Negative predictive value: $93.03 \%$

\section{Distribution of pathogens}

Out of 200 mid-stream urine samples, 36 (18.00\%) Gram-negative bacteria were isolated. Among them, Escherichia coli 29 (80.55\%) was predominant one followed by Enterobacter aerogenes 3(8.33\%), Pseudomonas aeruginosa 2(5.56\%), and Klebsiella pneumoniae 1 (2.78\%).

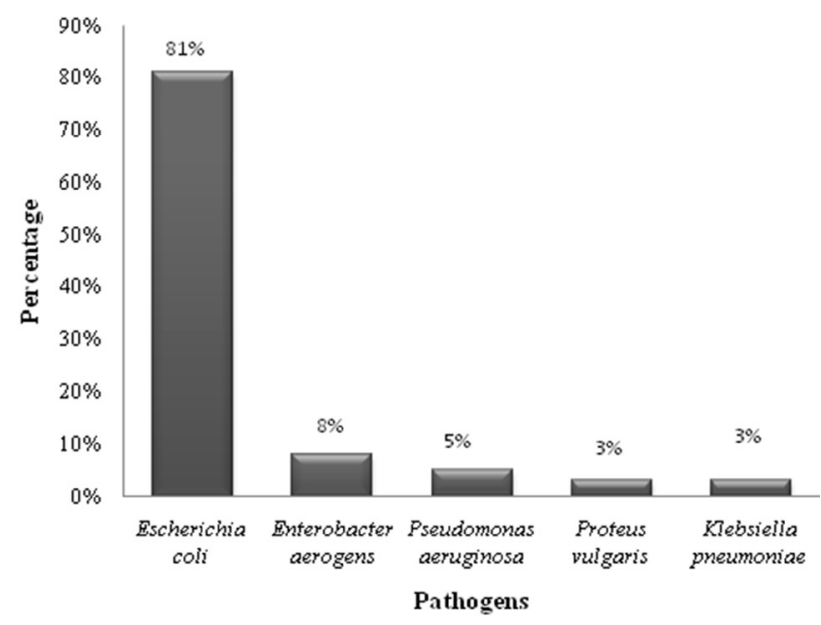

Figure 1. Distribution of pathogens

\section{Comparison of isolates between nitrite test and culture}

Escherichia coli 29(80.55\%) were predominant with $22(88.00 \%)$ nitrite test positive and $7(63.63 \%)$ nitrite test negative followed by Enterobacter aerogenes $3(8.33 \%)$ with $2(8.00 \%)$ nitrite test positive and $1(9.09 \%)$ nitrite test negative and Pseudomonas aeruginosa $2(5.56 \%)$ with $1(4.00 \%)$ positive and $1(9.09 \%)$ negative nitrite test. The least isolated were Klebsiella pneumoniae and Proteus vulgaris $(2.78 \%)$, both were nitrite test negative (Table 2$)$.

Table 2. Comparison of isolates between nitrite test and culture

\begin{tabular}{|l|c|c|c|}
\hline \multicolumn{1}{|c|}{ Bacteria isolated } & Frequency & Number of nitrite positive isolates & Number of nitrite negative isolates \\
\hline Escherichia coli & 29 & 22 & 7 \\
\hline Enterobacter aerogenes & 3 & 2 & 1 \\
\hline Pseudomonas aeruginosa & 2 & 1 & 1 \\
\hline Klebsiella pneumoniae & 1 & - & 1 \\
\hline Proteus vulgaris & 1 & - & 11 \\
\hline Total & 36 & 25 & 11 \\
\hline
\end{tabular}




\section{Antibiotic susceptibility pattern of the isolates}

Antibiotic susceptibility testing of the isolates revealed that isolates belonging to Enterobacteriaceae were susceptible to gentamicin (79.41\%) andamikacin (76.47\%). Antibiotics such as nalidixic acid (20.59\%), cefalexin (35.29\%) and amoxyclav (26.47\%) were least effective drugs. In case of $P$. aeruginosa, polymyxin B, colistin and ciprofloxacin were the most effective antibiotics. Among 36 isolates, 14 (38.89\%) were MDR of which $E$. coli $11(78.58 \%)$ were the predominant one. Two $(14.28 \%)$ isolates of E. aerogenes and a single isolate of K. pneumonia were MDR. Details of the antimicrobial patterns was shown in table 3. of UTIs. Also, the study determined common uropathogens among the UTI-suspected patients visiting Bharatpur hospital.

The sensitivity and the specificity of nitrite dipstick have been foundvaryingin different studies when compared to urine culture as the gold standard. In the current study, the sensitivity of urine nitrite dipstick test was $69.44 \%$ while specificity was higher $(89.63 \%)$. This was however unparallel to other studies where sensitivity between $27.3 \%$ to $43.6 \%$ and high specificity from $99.6 \%$ to $100 \%$ was reported. ${ }^{20,21}$ Another study conducted in India showed the sensitivity, specificity, Positive Predictive Value (PPV) and Negative Predictive Value (NPV) of nitrite test were $57.1 \%, 78.7 \%$,

Table 3. Antibiotic susceptibility pattern of the isolates

\begin{tabular}{|c|c|c|c|c|c|c|}
\hline \multirow{2}{*}{ Antibiotics } & \multicolumn{6}{|c|}{ Antibiotic susceptibility pattern } \\
\cline { 2 - 7 } & \multicolumn{2}{|c|}{ Enterobacteriaceae $\mathbf{n}=\mathbf{3 4}$ ) } & \multicolumn{2}{c|}{ P. aeruginosa (n = 2) } \\
\cline { 2 - 7 } & $\mathbf{S}$ & $\mathbf{I}$ & $\mathbf{R}$ & $\mathbf{S}$ & $\mathbf{I}$ & $\mathbf{R}$ \\
\hline Amikacin & $76.47 \%$ & $2.94 \%$ & $20.59 \%$ & $50.00 \%$ & $50.00 \%$ & $0.00 \%$ \\
\hline Amoxyclav & $26.47 \%$ & $23.53 \%$ & $50.00 \%$ & $0.00 \%$ & $0.00 \%$ & $100.00 \%$ \\
\hline Ciprofloxacin & $50.00 \%$ & $11.76 \%$ & $38.24 \%$ & $100.00 \%$ & $0.00 \%$ & $0.00 \%$ \\
\hline Cefixime & $26.47 \%$ & $11.76 \%$ & $61.76 \%$ & $0.00 \%$ & $0.00 \%$ & $100.00 \%$ \\
\hline Levofloxacin & $50.00 \%$ & $11.76 \%$ & $38.24 \%$ & $0.00 \%$ & $100.00 \%$ & $0.00 \%$ \\
\hline Nalidixic acid & $20.59 \%$ & $8.82 \%$ & $70.59 \%$ & NT & NT & NT \\
\hline Nitrofurantoin & $52.94 \%$ & $5.88 \%$ & $41.18 \%$ & $100.00 \%$ & $0.00 \%$ & $0.00 \%$ \\
\hline Cotrimoxazole & $41.18 \%$ & $52.94 \%$ & $5.88 \%$ & NT & NT & NT \\
\hline Gentamicin & $79.41 \%$ & $0.00 \%$ & $20.59 \%$ & $50.00 \%$ & $0.00 \%$ & $50.00 \%$ \\
\hline Cefalexin & $35.29 \%$ & $8.82 \%$ & $55.88 \%$ & $0.00 \%$ & $100.00 \%$ & $0.00 \%$ \\
\hline Polymyxin B & NT & NT & NT & $100.00 \%$ & $0.00 \%$ & $0.00 \%$ \\
\hline Colistin & NT & NT & NT & $100.00 \%$ & $0.00 \%$ & $0.00 \%$ \\
\hline
\end{tabular}

S: Sensitive, I: Intermediate, R: Resistant, NT: Not Tested

\section{DISCUSSION}

The present study was aimed at evaluating the efficacy of a rapid urine dipstick test in comparison to urine culture for the diagnosis
$42.7 \%$ and $86.8 \%$ respectively. ${ }^{22}$

This difference in the values may be because of the different sample populations in different studies like high-risk populations, gender, old age population and because of different brands of strips used for dipstick biochemical analysis. 
The variations in the results might also be attributed to improper techniques for collection or transportation to the laboratory, allowing the colonizing bacteria to multiply, which result in false positive nitrite test which may result in under treatment and as consequences could cause real damage or sepsis to the urinary tract system. Nitrite negative results may occur due to a low colony forming unit count or dilute urine. In addition, a nitrite test does not detect organisms unable to reduce nitrate to nitrite, such as Enterococci, Staphylococci species, Acinetobacter. Another disadvantage of the nitrite test is that the causative microorganism and its antibiotic susceptibility are not known. ${ }^{21,23}$

Among 36 bacterial isolates recovered, all were Gram-negative bacteria. The result of this study is in tune with the previous studies where the prevalence was between $95-99.30 \% .{ }^{24-}$ ${ }^{26}$ The reason behind obtaining the majority of Gram-negative isolates may bethem being the normal habitat of the colon and their descent to the urinary tracts, urinary bladder and kidney caused urinary tract infection. ${ }^{26}$

In this study, Escherichia coli (80.55\%) was found to be predominant followed by Enterobacter aerogenes (8.33\%), Pseudomonas aeruginosa (5.56\%), Proteus vulgaris and Klebsiella pneumoniae (2.78\%). Higher prevalence of E. coli in this study also resonates with the study done by Baral and co-workers. ${ }^{26}$ Bhansali and his colleagues also reported a higher percentage of $E$. coli $(56 \%)$ than other isolates. ${ }^{27}$ Likewise, a previous study done in Western Regional Hospital, Pokhara also reported E. coli (50\%) as the most predominant uropathogen. ${ }^{28}$

E. coli is far most common pathogen isolated from urinary tract infection (UTI) and frequently originated from patient's intestinal flora. There are many components or products called virulence factor that helps the E. coli to colonize the mucosal uroepithelium causing an inflammatory reaction and eventually helps to precede infection from the lower urinary tract to the renal cavities and tissues. The common virulence factors of E. coli are mainly two types; Surface virulence factors that are produced on the surface of the cell and exported factors those produced within the cell and exported to the site of action. Surface virulence factors are of fimbrial nature adhesions apparatus which includes Type 1 fimbriae, $P$ fimbriae, $S$ fimbriae, F1C fimbriae, curli, flagellum, capsule and lipopolysaccharide. Similarly, some of the exported virulence factors include alpha haemolysins of RTX family, Cytotoxic necrotizing factor I, Cytolysin A, Enterobactin etc. that helps E. coli to attach to the mucosal epithelium and tissue matrix, helps in motility, adaptation, bio-film production as well as cytokines induction. ${ }^{29}$

In this study, the second commonest pathogen was Pseudomonas aeruginosa which accounted for 5\% (2/36). A similar study also reported 5.01\% $P$. aeruginosa among total isolates. ${ }^{30}$ However, the result is in contrast with the study studied done in Nepal where the prevalence rate is less than $1 \%$. $^{25,31}$

This study found that gentamicin $(79.41 \%)$ and amikacin $(76.67 \%)$ were the drugs of choice for infection caused by Enterobacteriaceae. Similar study done in Western Nepal, reported parallel result as $70.40 \%$ Enterobacteriaceae were susceptible to gentamicin. ${ }^{32}$ This study showed $70.59 \%$ resistivity towards nalidixic acid which resembles the study done elsewhere. ${ }^{33,34}$ All isolates of $P$. aeruginosa were completely susceptible to ciprofloxacin, colistin and polymyxin B. Mostly resistance to fluoroquinolones could be the generalized use of fluoroquinolones in animal feed (especially in poultry), and the subsequent transmission of resistant strains from animals to humans. ${ }^{35}$ This study recorded $38.89 \% \mathrm{MDR}$ isolates. This rate is 
similar to the one done in Bharatpur hospital, in Nepal, which recorded 33.90\% MDR isolates, ${ }^{36}$ whereas Awasthi et al. (2015) reported a higher rate of MDR isolates $(42.9 \%)$ as compared to this study. ${ }^{1}$ The increasing rate of MDR may be attributed to excessive use of broad-spectrum antibiotics as observed in this study along within adherence to infection control measures. ${ }^{37}$

\section{CONCLUSIONS}

For bacterial growth on culture media by standard Laboratory techniques upto 48 hours are required which means that diagnosis is undecided for a longer duration which leads to delay in treatment. Although culture is the gold standard test for diagnosis of UTI, dipstick tests for the detection of nitrite in urine are sensitive and specific enough and can be used as a diagnostic test for detection of UTI in resourcepoor setup, where facility of culture is not available. Regular monitoring of susceptibility patterns of antibiotics along with their careful usage are advised to avoid the dissemination of the drug resistant isolates.

\section{ACKNOWLEDGEMENTS}

We are indebted to the Microbiology laboratory of Bharatpur hospital, its staff and the department of Microbiology, Birendra Multiple Campus for helping during the entirety of work in one way or the other.

\section{REFERENCES}

1. Awasthi TR, Pant ND, Dahal PR. Prevalence of multidrug resistant bacteria in causing community acquired urinary tract infection among the patients attending outpatient department of Seti Zonal Hospital, Dhangadi, Nepal. Nepal J Biotechnol . 2015 Dec 30;3(1):55-9. doi:10.3126/njb.v3i1.14232

2. Bjerklund Johansen TE, Naber KG. Urinary tract infections . Vol. 3, Antibiotics. MDPI AG; 2014. p. 375-7. doi:10.3390/antibiotics3030375

3. Bhatt C, Shrestha B, Khadka S, Swar S, Shah B, Pun K. Etiology of urinary tract infection and drug resistance cases of uropathogenes. J Kathmandu Med Coll. 2012 May 26;1(2):114-20. doi:10.3126/jkmc.v1i2.8150

4. Nicolle LE, Evans G, Laverdieve M, Phillips P, Quan C, Rotstein C. Complicated urinary tract infection in adults . Vol. 16, Canadian Journal of Infectious Diseases and Medical Microbiology. Hindawi Limited; 2005. p. 349-60. doi:10.1155/2005/385768

5. Foxman B. Epidemiology of urinary tract infections: Incidence, morbidity, and economic costs. Am J Med . 2002 Jul 8;113(1 SUPPL. 1):5-13. doi:10.1016/ S0002-9343(02)01054-9

6. Cheesbrough M. District Laboratory Practice in Tropic Countries Part 2. Cambridge University; 2007. 105-106 p.

7. Flores-Mireles AL, Walker JN, Caparon M, Hultgren SJ. Urinary tract infections: Epidemiology, mechanisms of infection and treatment options . Vol. 13, Nature Reviews Microbiology. Nature Publishing Group; 2015. p. 269-84. doi:10.1038/nrmicro3432

8. Perkins J, Perkins K, Vilke GM, Almazroua FY. Is culture-positive urinary tract infection in febrile children accurately identified by urine dipstick or microanalysis? J 
Emerg Med . 2012 Dec 1;43(6):1155-9. doi:10.1016/j.jemermed.2012.05.006

9. Hajar F, Taleb M, Aoun B, Shatila A. Dipstick urine analysis screening among asymptomatic school children. N Am J Med Sci . 2011 Apr;3(4):179-84. doi:10.4297/najms.2011.3179

10. Marahatta R, Dhungel B, Pradhan P, Rai S, Choudhury D. Asymptomatic bacteriurea among pregnant women visiting Nepal Medical College Teaching Hospital, Kathmandu, Nepal. Nepal Med Coll J. 2011 Jun 1;13:107-10.

11. Subedi M, Basnyat S, Acharya S. Urinary Tract Infection in Pregnancy and its Correlation with Nitrite Test. J Nepal Health Res Counc. 1970 Jan 1;7(2):80-3. doi:10.3126/jnhrc.v7i2.3011

12. Diviney J, Jaswon MS. Urine collection methods and dipstick testing in nontoilet-trained children. Pediatr Nephrol 2020 367. 2020 Sep 12;36(7):1697-708. doi:10.1007/S00467-020-04742-W

13. Williams GJ, Macaskill P, Chan SF, Turner RM, Hodson E, Craig JC. Absolute and relative accuracy of rapid urine tests for urinary tract infection in children: a meta-analysis . Vol. 10, The Lancet Infectious Diseases. Elsevier; 2010. p. 240-50. doi:10.1016/S14733099(10)70031-1

14. Mori R, Yonemoto N, Fitzgerald A, Tullus K, Verrier-Jones K, Lakhanpaul M. Diagnostic performance of urine dipstick testing in children with suspected UTI: A systematic review of relationship with age and comparison with microscopy. Acta Paediatr Int J Paediatr . 2010 Apr;99(4):581-4. doi:10.1111/j.1651-2227.2009.01644.x

15. Prestinaci F, Pezzotti P, Pantosti A. Antimicrobial resistance: a global multifaceted phenomenon. Pathog Glob Health . 2015 Oct 1;109(7):309. doi :10.1179/2047773215Y.0000000030

16. Biswas D, Gupta P, Prasad R, Singh V, Arya M, Kumar A. Choice of antibiotic for empirical therapy of acute cystitis in a setting of high antimicrobial resistance. Indian J Med Sci . 2006 Feb 1;60(2):53-8. doi:10.4103/00195359.19913

17. Rijal A, Ghimire G, Gautam K, Barakoti A. Antibiotic susceptibility of organisms causing urinary tract infection in patients presenting to a teaching hospital. J Nepal Health Res Counc. 2012;10(1):24-7.

18. Clinical and Laboratory Standards Institute. Performance standards for antimicrobial susceptibility testing. CLSI. Pennsylvania, USA. 24th informational Suppl (M100-S28) Wayne. 2016;

19. Magiorakos AP, Srinivasan A, Carey RB, Carmeli Y, Falagas ME, et al. Multidrug-resistant, extensively drug-resistant and pandrug-resistant bacteria: an international expert proposal for interim standard definitions for acquired resistance. Clin Microbiol Infect . 2012;18(3):268-81.

20. Buzayan MM, Tobgi RS. Comparison of urine culture, microscopy and nitrite dipstick tests in the detection of urinary tract infection. Vol. 20, Journal of the Bahrain Medical Society. 2008. p. 124-7.

21. Eidelman Y, Raveh D, Yinnon AM, 
Ballin J, Rudensky B, Gottehrer NP. Reagent strip diagnosis of UTI in a high-risk population. Am J Emerg Med. 2002 Mar 1;20(2):112-3.doi: 10.1053/ ajem.2002.31145

22. Taneja N, Chatterjee $\mathrm{S}$, Singh $\mathrm{M}$, Sivapriya S, Sharma M, Sharma S. Validity of quantitative unspun urine microscopy, dipstick test leucocyte esterase and nitrite tests in rapidly diagnosing urinary tract infections. J Assoc Physicians India. 2010 Aug 1;58:485-7.

23. Koeijers JJ, Kessels AGH, Nys S, Bartelds A, Donker G, Stobberingh $\mathrm{EE}$, et al. Evaluation of the nitrite and leukocyte esterase activity tests for the diagnosis of acute symptomatic urinary tract infection in men. Clin Infect Dis . 2007;45(7):894-6. doi:10.1086/521254

24. Acharya A, Gautam R, Subedee L. Uropathogens and their antimicrobial susceptibility pattern in Bharatpur, Nepal. Nepal Med Coll J. 2011 Mar;13(1):30-3.

25. Rai G, Upreti H, Rai S, Shah K, Shrestha R. Causative agents of urinary tract infections in children and their antibiotic sensitivity pattern: a hospital based study. Nepal Med Coll J. 2008 Jul 1;10:86-90.

26. Baral P, Neupane S, Marasini B, Ghimire K, Lekhak B, Shrestha B. High prevalence of multidrug resistance in bacterial uropathogens from Kathmandu, Nepal. BMC Res Notes . 2012;5(38). doi:10.1186/1756-0500-5-38

27. Bhansali A, Inbaraj L, George C, Norman G. Can urine dipstick test be an alternative to detect urinary tract infection in limited resource setting? A validity study from Bangalore, India. J Fam Med Prim Care .2020;9(2):561. doi:10.4103/jfmpc.jfmpc_696_19

28. Khadka KS, Khadka J, Lekhak B, Shrestha P, Raj Tiwari B. Incidence of Urinary Tract Infection among the Patients Visiting Western Regional Hospital , Pokhara, Nepal. J Heal Allied Sci . 2012 Nov 26;2(1):35-7. doi:10.37107/jhas.73

29. Emody L, Kerényi M, Nagy G. Virulence factors of uropathogenic Escherichia coli. Int J Antimicrob Agents . 2003 Oct 1;22(SUPPL. 2). doi:10.1016/S09248579(03)00236-X

30. Kattel HP, Mishra SK, Acharya J, Singh AS, Rijal BP, Pokhrel BM. Relationship Between Pyuria and Bacteriuria in Suspected Urinary Tract Infection. J Nepal Assoc Med Lab Sci. 2009;10(April 2016):19-21.

31. Thapa P, Parajuli K, Poudel A, Thapa A, Manandhar B, Laudari D, et al. Causative Agents and Susceptibility of Antimicrobials among Suspected Females with Urinary Tract Infection in Tertiary Care Hospitals of Western Nepal. J Chitwan Med Coll . 2013 Aug 13;3(2):16-9. doi:10.3126/jcmc. v3i2.8436

32. Das RN, Chandrashekhar TS, Joshi HS, Gurung M, Shrestha N, Shivananda PG. Frequency and susceptibility profile of pathogens causing urinary tract infections at a tertiary care hospital in western Nepal. Singapore Med J. 2006 Apr;47(4):281-5. 
33. Rashedmarandi F, Rahnamayefarzami M, Saremi M, Sabouri R. A Survey On Urinary Pathogens And tmlTheir Antimicrobial Susceptibility Among Patients With Significant Bacteriuria. Iran J Pathol . 2008 Sep 1;3(4):191-6.

34. Behrooozi A, Rahbar M, V J, Yousefi. A survey on epidemiology of urinary tract infections and resistance pattern of uropathogens in an Iranian 1000bed tertiary care hospital. African J Microbiol Res . 2010 May 4;4(9):753-6. doi:10.5897/AJMR.9000015

35. Miller LG, Tang AW. Treatment of Uncomplicated Urinary Tract Infections in an Era of Increasing Antimicrobial Resistance. Mayo Clin Proc. 2004 Aug
1;79(8):1048-54. doi:10.4065/79.8.1048

36. Adhikari S, Khadka S, Sapkota S, Rana JC, Khanal S, Neupane A, et al. Prevalence and Antibiograms of Uropathogens from the Suspected Cases of Urinary Tract Infections in Bharatpur Hospital, Nepal. J Coll Med Sci. 2019;15(4):260-6. doi: 10.3126/ jcmsn.v15i4.20856

37. Siwakoti S, Subedi A, Sharma A, Baral R, Bhattarai NR, Khanal B. Incidence and outcomes of multidrug-resistant gramnegative bacteria infections in intensive care unit from Nepal- a prospective cohort study. Antimicrob Resist Infect Control .2018;7:114. doi:10.1186/s13756018-0404-3

Citation: Adhikari A, Sapkota S, Poudel P, Regmi R, Kafle S, Baral S, Dallakoti N, Rana J, Mahato B, Rijal K. Comparison of Urine Dipstick Nitrite Test with Urine Culture in the Diagnosis of Urinary Tract Infection. JCMS Nepal. 2021; $17(4) ; 331-40$. 